\title{
Electronic Nicotine Delivery Device (ENDS)
}

\author{
Robert West ${ }^{1}$ \\ 1 Univers ity College London, University of London
}

An electronic hand-held vaping device that produces for inhalation by a person an aerosol containing nicotine formed by heating a liquid, called an e-liquid. The e-liquid contains nicotine and one or more humectants (propylene glycol, glycerol or glycerin), usually together with flavourants. The heating is achieved by means of a battery-powered heating element that is activated by a switch or by suction as the user sucks on the mouthpiece of the device. 